\title{
NUEVA GESTIÓN PÚBLICA Y EDUCACIÓN: ELEMENTOS TEÓRICOS Y CONCEPTUALES PARA EL ESTUDIO DE UN MODELO DE REFORMA EDUCATIVA GLOBAL
}

\author{
APRESENTAÇÃO
}

Palabras clave: Nueva gestión pública (NGP); Globalización; Mercantilización; Evaluación basada en resultados; Reforma educativa; Difusión internacional de políticas; Re-contextualización; Convergencia; Rendición de cuentas; Autonomía escolar; Estándares curriculares comunes; Financiación competitiva.

\section{Introducción ${ }^{1}$}

$\mathrm{L}$

a nueva gestión pública (NGP) es un programa de reforma del sector público que aplica conocimientos e instrumentos de la gestión empresarial y de disciplinas afines, y que tiene como finalidad "[...] mejorar la eficiencia, la eficacia y el rendimiento general de los servicios públicos en las burocracias modernas [...]". (VIGODA, 2003, p. 813) El desarrollo de reformas de NGP implica desagregar de forma más marcada las funciones de proveedores y usuarios, fragmentar los servicios públicos en unidades de gestión más independientes o autónomas, y promover una gestión basada en la obtención de resultados tangibles. (KALIMULLAH et al., 2012)

En los últimos años, la NGP ha penetrado con fuerza en la agenda educativa global. Muchos gobiernos, tanto de países industrializados como en vías de desarrollo, han introducido medidas de NGP en uno o más sectores, incluyendo el educativo. (HOOD; PETERS, 2004; CHRISTENSEN; LÆGREID, 2011) El hecho de que la educación sea uno de los sectores de la administración pública con 
mayor dotación presupuestaria y de personal, hace que este sector sea especialmente proclive a la aplicación de reformas orientadas por la NGP. En aquellos lugares donde se ha aplicado, la NGP ha alterado de manera drástica la forma como se concibe la gobernanza de las instituciones educativas, ya que principios como la autonomía escolar, la rendición de cuentas, las gestión basada en resultados o la libertad de elección escolar han penetrado profundamente en cómo se regulan, proveen y financian los servicios educativos. (TOLOFARI, 2005)

Los motivos y las lógicas por las que se adoptan la propuesta de la NGP en el campo educativo, así como la manera como dicha propuesta se recontextualiza en marcos regulatorios y programas educativos concretos varía sustancialmente en distintos países y regiones. La NGP no es un modelo de reforma educativa monolítico ni adopta la misma forma en todos los lugares; más bien, se puede concretar en enfoques de gobernanza y en diseños de políticas educativas bien diferenciados. Por ejemplo, como desarrollaremos más adelante, las políticas de autonomía escolar varían en función de si se centran en dar más poder a las escuelas a nivel curricular, a nivel organizativo o a nivel de gestión de recursos. (OCDE, 2011) Algo parecido sucede con las medidas de rendición de cuentas en educación que, entre otros factores, varían según el nivel de estandarización educativa que implican, de quién es el 'principal' y quién es el 'agente' de la rendición de cuentas, y de las consecuencias que conllevan para el personal docente y/o las escuelas objeto de la evaluación. (MAROY; VOISIN, 2013)

Este monográfico explora en profundidad la difusión internacional y la re-contextualización de la NGP en realidades educativas concretas. Entre otras cuestiones, los artículos que conforman este monográfico analizan cómo y por qué la NGP ha sido adoptada y promulgada en diferentes territorios, así como los factores institucionales y políticos que median en la re-contextualización de la NGP en el sector educativo. Concretamente, los artículos se centran los casos de Brasil, la provincia de Buenos Aires, Cataluña, Chile, Francia, Inglaterra, Italia, Noruega y Quebec, los cuales han sido escogidos dada la centralidad que la NGP ha adquirido en los sistemas educativos de cada uno de estos territorios. Por lo que respecta a los autores, Dalila Andrade se ha centrado en el caso de Brasil; Ma Fernanda Astiz en el caso de la Provincia de Buenos Aires; Antoni Verger, Marta Curran y Lluís Parcerisa en el caso de Cataluña; Alejandra Falabella en el caso de Chile; Jean-Luis Derouet, Romuald Normand y Ramón Pacheco en el caso de Francia; Dave Hall y Hellen Gunter en el caso de Inglaterra; Emiliano Grimaldi, Roberto Serpieri y Danilo Taglietti en el caso de Italia; Jorunn Moller y Guri Skedsmo en el caso de Noruega; y Christian Maroy, Cécile Mathou, Samuel Vaillancourt y Annelise Voisin en el caso de Quebec.

Este artículo introductorio tiene por objetivo explorar la relación entre la NGP y la política educativa, así como aportar elementos teóricos y conceptuales al estudio de dicha temática. Se estructura en tres secciones principales. En la 
primera sección, se enumeran los principios que orientan la NGP y el amplio abanico de políticas educativas en que se traducen dichos principios. Entre otras cuestiones, veremos que muchas de las políticas educativas inspiradas en la NGP tienen significados e implicaciones políticas bien diferenciados.

En la segunda sección se identifican un conjunto de mecanismos que explican por qué y cómo los países deciden adoptar las ideas de la NGP en procesos de reforma educativa. Nos referimos a factores y mecanismos como la ideología política, los legados institucionales, la legitimación externa y los marcos de significado. En la tercera y última sección reflexionamos sobre cómo las políticas de la NGP en el campo educativo pueden ser traducidas y recontextualizadas de forma múltiple. En esta sección apuntamos también algunas de las paradojas más recurrentes en la aplicación de este tipo de políticas en el campo educativo. Muchas de las aportaciones teóricas que contiene este artículo son ilustradas con ejemplos concretos que provienen de los estudios de caso incluidos en este monográfico sobre NGP y política educativa.

\section{Principios y políticas de la NGP en educación}

El programa de la NGP no implica una retirada del Estado del ámbito de los servicios públicos, sino que supone más bien un replanteamiento de las funciones gubernamentales en la gestión de dichos servicios. (HUDSON, 2007) Desde la óptica de la NGP se espera del Estado que, más que proveer servicios públicos de manera directa, fortalezca su rol de regulador, evaluador y distribuidor de incentivos a los proveedores autónomos de dichos servicios. De acuerdo a la famosa metáfora de Osborne y Gaebler (1993), el Estado debería centrarse en guiar (steering) en lugar de remar (rowing) los servicios públicos. Según Hood (1991), los principios fundamentales por los que se rige la NGP son:

Gestión profesional en el sector público; establecimiento de estándares y medidas de desempeño explícitas; mayor énfasis en el control de resultados; desagregar el sector público en unidades más pequeñas; mayor dosis de competencia en el sector público; énfasis en estilos de gestión provenientes del sector privado; énfasis en una mayor disciplina y mesura en el uso de recursos. (HOOD, 1991, p. 4-5)

Estos principios, una vez transpuestos al campo de la política educativa, cristalizan en un amplio abanico de políticas como las que incluimos en la Tabla 1. 


\section{Tabla 1}

Principios de la NGP y política educativa

\begin{tabular}{|c|c|}
\hline PRINCIPIOS NGP & POLÍTICAS EDUCATIVAS \\
\hline $\begin{array}{l}\text { Gestión profesional de los } \\
\text { servicios públicos }\end{array}$ & $\begin{array}{l}\text { - Profesionalización y empoderamiento } \\
\text { de los directores de escuela }\end{array}$ \\
\hline $\begin{array}{l}\text { Normas y medidas de } \\
\text { desempeño más explícitas }\end{array}$ & $\begin{array}{l}\text { - Definición de indicadores de calidad y } \\
\text { de benchmarks sobre "éxito" educativo. } \\
\text { - Estándares curriculares comunes }\end{array}$ \\
\hline $\begin{array}{l}\text { Énfasis en el control de } \\
\text { resultados }\end{array}$ & $\begin{array}{l}\text { - Evaluación externa de los resultados y } \\
\text { del rendimiento escolar }\end{array}$ \\
\hline $\begin{array}{l}\text { Desagregar el sector público en } \\
\text { unidades de gestión más } \\
\text { pequeñas }\end{array}$ & $\begin{array}{l}\text { - Autonomía escolar, school-based } \\
\text { management }\end{array}$ \\
\hline $\begin{array}{l}\text { Mayor competición en el sector } \\
\text { público }\end{array}$ & $\begin{array}{l}\text { - Subsidios públicos a las escuelas } \\
\text { privadas } \\
\text { - Financiación per cápita } \\
\text { - Publicación de los resultados obtenidos } \\
\text { por las escuelas en pruebas } \\
\text { estandarizadas }\end{array}$ \\
\hline $\begin{array}{l}\text { Emular el estilo gerencial del } \\
\text { sector privado }\end{array}$ & $\begin{array}{l}\text { - Flexibilidad de la escuela en la } \\
\text { contratación y el despido } \\
\text { - Estilos gerenciales de dirección de } \\
\text { escuela }\end{array}$ \\
\hline $\begin{array}{l}\text { Mayor disciplina/ parsimonia en } \\
\text { el uso de recursos }\end{array}$ & $\begin{array}{l}\text { - Financiación de las escuelas en base a } \\
\text { resultados } \\
\text { - Remuneración docente basada en } \\
\text { criterios de mérito/productividad }\end{array}$ \\
\hline
\end{tabular}

Fuente: Adaptación de Gunter y Fitzgerald (2013).

Ahora bien, a pesar de contar con una serie de principios comunes, la NGP se puede concretar en 'configuraciones de políticas' muy diversas. De hecho, los postulados de la NGP son a menudo ambiguos e incluyen medidas que no tienen por qué tener una connotación unívoca ni necesariamente pro-mercado o neoliberal. Este es el caso, por ejemplo, de la 'autonomía escolar', que es una de las medidas más emblemáticas de las reformas de NGP en el campo educativo dada la importancia que la NGP concede a la fragmentación de los servicios públicos en unidades de gestión más independientes. Bajo la etiqueta de autonomía escolar, programas y prácticas de una naturaleza muy distinta pueden ser emplazados. Por un lado, existe un enfoque gerencialista de la autonomía escolar, conocido en el mundo anglosajón como school-based management, que implica una descentralización de las responsabilidades de gestión a nivel de escuela, las cuales acostumbran 
a recaer en el equipo directivo. Como si de un gestor de una empresa privada se tratara, el director pasa a contar con más competencias para administrar los recursos económicos y humanos, conseguir financiación externa, y contratar y despedir al personal docente. (GUNTER; FORRESTER, 2009) Desde esta perspectiva, la autonomía escolar otorga mayor poder al director de la escuela en detrimento del poder de los maestros, y corre el riesgo de segmentar el sistema educativo en el sentido de que algunas escuelas pueden generar más recursos externos que otras. (EURYDICE, 2007)

Por otro lado, la autonomía escolar tiene también una connotación más bien educativa y pedagógica que consiste en otorgar a las escuelas más capacidad de decisión respecto el proyecto educativo, los contenidos curriculares que se imparten y los sistemas de evaluación que se aplican. El objetivo de esta vertiente de la autonomía consiste en promover que la educación escolar se adapte a las particularidades y necesidades del contexto social y cultural en el que las escuelas están ubicadas. En el marco de este planteamiento, la autonomía escolar implica la profesionalización docente, ya que se concibe a los maestros como profesionales autónomos con capacidad de decidir acerca de los principales componentes del proceso de enseñanza-aprendizaje. (SLEEGERS; WESSELINGH, 1995) Así, en contraste con el modelo gerencialista, la autonomía escolar se asocia en este caso con principios más bien progresistas como son la profesionalización, la renovación pedagógica y la atención a la diversidad. En cualquier caso, ambos 'tipos ideales' de autonomía escolar (la autonomía de gestión y la pedagógica) se acostumbran a dar de forma híbrida en realidades educativas concretas. (VERGER; CURRAN, 2014)

Reflexiones similares sobre la naturaleza dual y la variedad de significados de las políticas educativas se podrían hacer en relación a la rendición de cuentas, que es otro de los instrumentos principales de la NGP en el campo educativo. La rendición de cuentas, o accountability, responde a una lógica de gobernanza escolar centrada en el desempeño y en relaciones profesionales auditables y performativas. (POWER, 1997; BALL, 2001) Esta política somete la labor docente y sus objetivos a medidas estandarizadas de calidad y, por ello, representa un desafío a la autonomía profesional de los maestros. La promoción de cuasi-mercados, el aumento de la competitividad entre escuelas y el énfasis en el desempeño escolar, sustentado por el desarrollo de sistemas de información, han fortalecido que las políticas de rendición de cuentas acaben contribuyendo a promover la elección de centro. La NGP ha estimulado el mayor alcance político de la evaluación educativa, y la mayor presencia de planes estratégicos e indicadores de desempeño en base a los que asignar recursos a los centros. Las condiciones de trabajo y los salarios del profesorado están también cada vez más sujetos a nuevos regímenes de rendición de cuentas en muchos lugares del mundo. Stephen Ball (2003) muestra cómo determinadas medidas de rendición de cuentas han fortalecido el control y la presión sobre la profesión docente. 
Ahora bien, como decíamos en relación a las políticas de autonomía escolar, la rendición de cuentas se puede concretar en modelos y aproximaciones muy diferentes. Este hecho es tenido en cuenta por organizaciones internacionales como la OCDE que, en lugar de la tradicional rendición de cuentas vertical e intrusiva, propone la implementación de una accountability horizontal en la que el cuerpo profesional docente juegue un papel importante tanto en la definición como en la implementación de la política. (OCDE, 2013) De esta manera se promueve una rendición de cuentas que, además de incluir información sobre desempeño escolar obtenida en evaluaciones centralizadas, genere otro tipo de datos desde otro tipo de fuentes (incluyendo a las familias y a representantes de la comunidad educativa). Se espera, por lo tanto, que estas evaluaciones acaben teniendo unas connotaciones más formativas que sumativas.

En el último apartado de este artículo, discutimos en más detalle las diferentes configuraciones de las políticas de NGP en educación en base a los estudios de caso incluidos en este monográfico.

\section{La adopción de la NGP en los procesos de reforma educativa: lógicas y mecanismos clave}

La NGP, como cualquier otro modelo de reforma educativa global, no ha sido uniformemente recibido ni adoptado en los distintos lugares en los que ha penetrado. Aunque las reformas globales en educación presentan características comunes en distintas partes del mundo, el conglomerado de políticas en el que acaban resultando se encuentra fuertemente mediado por, entre otras contingencias, factores de origen histórico, político-administrativo e cultural, así como por la compleja interacción entre fuerzas globales y locales (VERGER et al., 2012). Según Peck y Theodore (2010, p. 170),

[...] las políticas globales mutan durante sus travesías, y rara vez viajan como paquetes completos, sino que se desplazan de forma parcial - como discursos selectivos, ideas inconclusas y modelos sintetizados -, llegando a su destino no como copias, sino que en forma de políticas en plena transformación.

De forma similar, Ball (1998) considera que las políticas globales son raramente traducidas al pie de la letra en prácticas locales dado que, más allá de un programa pre-fijado, éstas son parte de un debate técnico y político muy disputado, cuyos resultados son contingentes a las diferentes realidades locales.

Los contextos locales y, en particular, los agentes que operan en esos contextos, son estratégicamente selectivos. Actores estatales y no estatales que 
operan a escala local tienen la capacidad de manejar y aportar significados específicos a las ideas globales, y las tienden a recombinar con políticas y prácticas locales existentes, así como con sus propias experiencias previas. (CAMPBELL, 2004) Además, los actores locales pueden también instrumentalizar las agendas y políticas globales para acercarlos a (y legitimar) sus preferencias de políticas y/o intereses políticos específicos.

En este apartado exploramos las razones por las que la NGP ha sido adoptada en un amplio conjunto de países y territorios que son muy diferentes entre sí - de hecho, los casos analizados en el presente volumen presentan importantes diferencias en los planos cultural, socioeconómico e institucional. Con este objetivo en mente, y en base a diferentes corrientes teóricas, revisaremos una variedad de lógicas y mecanismos que explican la adopción de la NGP en el campo educativo. Estas herramientas teóricas y conceptuales contribuirán a matizar las aproximaciones más estructuralistas a la globalización de la política educativa. En otras palabras, dichas herramientas nos ayudarán a entender no sólo por qué las ideas de la NGP se han difundido en diferentes realidades educativas, sino también por qué la NGP no ha convergido en un único enfoque mundial de reforma educativa. En líneas generales, los mecanismos identificados ofrecen explicaciones diferentes, aunque no necesariamente excluyentes, de por qué los gobiernos adoptan modelos educativos globales, como el de la NGP, y de cómo los transforman y adaptan a sus propias realidades.

\subsection{Ideología política: la socialdemocracia se suma a la NGP}

A pesar de que, a lo largo del siglo XX, se han producido diferentes oleadas de reformas en la gestión del sector público, muchos autores consideran que la NGP empezó a penetrar en los países industrializados occidentales en la década de los ochenta. Entre los países precursores de la NGP, encontramos Estados Unidos, el Reino Unido y Canadá, todos ellos gobernados por partidos conservadores en ese periodo. (TOLOFARI, 2005) Los gobiernos conservadores justificaron la implantación de reformas de NGP con un fuerte componente pro-mercado por la necesidad de promover una mayor eficiencia en el sector público en un periodo de austeridad económica y fiscal. Otro incentivo para la nueva derecha a la hora de promover la NGP fue el de debilitar el poder de los sindicatos y de otros grupos de presión a través de la fragmentación de los servicios públicos.

Posteriormente, aunque por motivos similares, instituciones financieras internacionales como el Banco Mundial y el Fondo Monetario Internacional difundieron los postulados de la NGP en países en vías de desarrollo. En los años noventa, la NGP se convirtió en un componente clave en los programas de reajuste estructural promovidos por dichas organizaciones que consideraban que, 
para que las medidas de estabilidad macroeconómica que recetaban a los países fueran efectivas, éstas deberían combinarse con un enfoque de la elección pública y gerencialista en la reforma del sector público. (FINE, 2006)

Por lo tanto, aparentemente, en los primeros años de la NGP, la motivación presupuestaria era el principal motor de difusión y adopción, tanto en los países desarrollados como en los países en vías de desarrollo. (HENDRIKS; TOPS, 2003) Sin embargo, investigaciones más recientes señalan que, en algunos casos, la adopción de la NGP no tiene por qué ir necesariamente vinculada a periodos de crisis económica ni a la ideología neoliberal, sino que otras motivaciones están también en juego. Para Klitgaard (2007) y Wiborg (2013), las disputas entre los partidos políticos mayoritarios y, en concreto, la evolución del pensamiento socialdemócrata en Europa, son elementos centrales a la hora de comprender la adopción de la NGP en los países escandinavos. Estos autores observan que los gobiernos socialdemócratas de esta región adoptaron ideas de reforma del sector público que originalmente provenían de la derecha, con el objetivo de "modernizar" la educación pública y otros servicios públicos en un contexto de crisis de legitimidad de los Estados de bienestar. Dicha modernización debería conllevar la diferenciación de los servicios y la oferta de más opciones para los usuarios. En definitiva, los socialdemócratas vieron en la NGP un instrumento con el que transformar, a la vez que proteger, la idea de "Estado de bienestar universal" que, en cierto modo, era su arma política y electoral más valiosa.

Los gobiernos socialdemócratas deciden introducir reformas cuando la élite del partido percibe que determinados problemas de políticas devienen una amenaza para la legitimidad del Estado de bienestar universal. Las instituciones políticas, es decir, las políticas de bienestar, que funcionan como recursos de poder, tienen que ser legítimas ya que de lo contrario pueden ir en contra de sus intereses políticos básicos. (KLITGAARD, 2007, p. 172)

No obstante, este proceso de adopción de la NGP no sólo se ha dado en los países escandinavos. Por ejemplo, bajo una lógica modernizadora similar, el partido laborista británico promovió reformas en el sector público basadas en la NGP cuando formó gobierno a finales de la década de los 1990. (MCLAUGHLIN et al., 2001; véase también VERGER; CURRAN; PARCERISA en este volumen para el caso catalán)

\subsection{Legados: NPM, taylorismo y burocracia}

La NGP es una tendencia modernizadora en la administración pública, rupturista respecto a las regulaciones taylorista y burocrática. En el contexto del 
Reino Unido, David Hall y Helen Gunter (en este volumen) demuestran cómo el surgimiento de la NGP desplazó el bienestarismo (o welfarsim) de posguerra y reestructuró la educación pública poniendo más énfasis en principios como la elección, los mercados y la medida del desempeño educativo. Esta búsqueda de la eficiencia y la efectividad escolar, que se encuentra en el núcleo de los discursos justificadores de muchas reformas educativas actuales, no es nueva. Durante los años veinte, en los EEUU, la gestión de la eficiencia ganó interés entre los políticos locales asesorados por expertos especializados en la metodología de los test del coeficiente intelectual y la gestión científica implementada por Ralph Winslow Taylor en el sector industrial. (CALLAHAN, 1962) Construidas como redes de administradores, y apoyadas por potentes organizaciones profesionales, estos agentes difundieron de forma exitosa lo que ellos consideraban la "mejor manera" de llevar a cabo reformas educativas orientadas a la mejora de calificaciones y resultados del alumnado. (TYACK; HANSOT, 1982) Estos expertos en administración proponían nuevas formas de organización escolar efectiva inspirándose en la nueva división del trabajo en la industria y el sector del ferrocarril. Para ello, promovían la adopción de estándares para la selección de estudiantes y la gestión escolar.

Esta racionalización administrativa hacia la eficiencia se mantuvo como un discurso dominante conforme la urbanización y la industrialización creaban nuevos problemas sociales en forma de inmigración, pobreza, y falta de educación y de sanidad. En este contexto, la burocratización de los sistemas educativos era percibida como un imperativo económico: la escolarización de los jóvenes tenía que desarrollar una "nueva inteligencia" con la que dar respuesta a las necesidades industriales emergentes. El ideal del gobierno consistía en una sociedad planeada y liderada por expertos y administradores científicos. Los defensores de la gestión científica y sus aliados luchaban contra políticos locales de corte tradicional para imponer una nueva concepción de la administración escolar basada en pruebas objetivas (testing), un régimen que en la actualidad es predominante en países como los EEUU y que fue impulsado con las políticas de rendición de cuentas de los años ochenta.

En Europa, salvo en el Reino Unido, el testing no era concebido inicialmente como un instrumento necesariamente intrusivo. El desarrollo de la administración escolar tomó diferentes vías a través de una racionalización burocrática de parte del Estado. (CLARKE; NEWMAN, 1997) La ambicionada efectividad educativa se lograba a través de planteamientos educativos y curriculares basados en acciones rutinarias y predecibles. El rol de la administración educativa (sustentado por reglas y controles) era el de transformar tareas complejas en dispositivos estables, tales como los exámenes y los horarios. La administración aspiraba a tratar a cada alumno justamente y de acuerdo a reglas y procedimientos implementados con igualdad y neutralidad. Esta forma de regulación era una 
alternativa al clientelismo, la corrupción y el nepotismo que predominaban antes de la aparición del sistema educativo moderno y obligatorio. La nueva administración burocrática garantizaba un nuevo gobierno escolar, y el trato igualitario de los alumnos. Los principios de la burocracia aseguraron que la política escolar fuera protegida de pasiones e intereses de la sociedad civil.

Pero la administración burocrática no era suficiente, y la acción pública tuvo que ser apoyada por expertos capacitados que aportaran soluciones a los problemas sociales y políticos. El profesionalismo fue así el segundo de los legados desarrollados con la evolución de los sistemas administrativos. En el campo educativo, al profesor profesional se le exigía el servicio al bien público, así como un cierto compromiso con la escolarización obligatoria y la democratización de la educación. Su posición como experto y su conocimiento eran considerados un medio para sustentar la inversión en capital humano y la construcción de ciudadanía. Enmarcados en códigos profesionales y éticos, los maestros eran llamados a definir un ethos de servicio a los valores universales y al interés público, que sería legitimado y transmitido por organizaciones profesionales. (EVETTS, 2003) Este profesionalismo implicaba confianza y solidaridad en las relaciones profesionales. Se basaba en la autonomía y en regulaciones entre iguales. Dependía de un sistema común de instrucción y contratación, una socialización a largo plazo, y una cultura e identidad profesional específica. Estas características de la profesión docente son hoy día desafiadas por la NGP, en un momento en el que la efectividad y los resultados de aprendizaje se han convertido en el principal objetivo de las políticas educativas.

El nivel de profesionalización docente de un país, así como otros tipos de legados institucionales, puede dificultar, matizar o retardar la adopción de políticas basadas en la NGP en el sector educativo. Por ejemplo, en Noruega prevalece una fuerte tradición de 'escuela comprehensiva' que, según Moller y Skedsmo (en este volumen), "[...] va en contra de algunos elementos enfatizados en las reformas de NGP, como la privatización, la competencia y el mercado [...]”. De manera similar, en Francia, la separación entre Estado e intereses privados, y la desconfianza en las ideas relacionadas con el gerencialismo, ha impedido el desarrollo de la elección de escuela, la autonomía y el mercado en educación (véase DEROUET; NORMAND; PACHECO en este número). La NGP se asocia, en este país, a la tradición napoleónica, y los cuerpos profesionales e instituciones del Estado controlan la implementación de las reformas educativas. El apego a la igualdad de oportunidades, que se encuentra en el núcleo del ideario de la sociedad francesa, limita los intentos de diversificación y flexibilización del sector educativo.

En Italia, la administración pública, incluso en momentos de mucho cuestionamiento, mantiene algunos rasgos napoleónicos (legalismo, centralismo, burocracia) y el Ministro de Educación mantiene el control de la gobernanza general del sistema a pesar de la descentralización y de la mayor autonomía escolar 
otorgada a nivel local (GRIMALDI; SERPIERI; TAGLIETTI en este número). Esta centralidad del Estado es también una característica del sistema educativo de Quebec, como Christian Maroy y sus colegas explican en este volumen. En Quebec, la NGP y la accountability se encuentran entremezclados con un legado burocrático y centralizado, pero también con una tradición de descentralización y de participación de las comunidades locales en el campo educativo.

Curiosamente, en Chile encontramos un ejemplo atípico de cómo los legados históricos condicionan la adopción de la NGP. Mientras que la NGP es concebida en la mayoría de casos como una herramienta para desburocratizar y, en cierta manera, mercantilizar los sistemas de educación pública, en Chile se adoptaron medidas de NGP y sistemas de incentivos en los 1990 para intentar revertir las desigualdades que la drástica reforma pro-mercado adoptada durante los 1980 generó en el sistema educativo (véase Falabella en este volumen).

\subsection{Legitimación exterior: la 'Cultura Mundial' y el rol de las organizaciones internacionales}

En la mayoría de las ocasiones, los procesos de adopción de la NGP se encuentran fuertemente condicionados por el legado institucional de un país. Sin embargo, en muchos países, la adopción de la NGP no está necesariamente fundamentada sobre trayectorias históricas y/o legados administrativos concretos, sino que es el resultado de presiones externas recibidas por los estados. Como argumentan los teóricos neo-institucionalistas y, en concreto, aquellos que se adscriben a la Teoría de la Cultura Mundial, las concepciones del Estado moderno - construidas normativa y culturalmente - explican una parte importante de la difusión transnacional de un amplio abanico de reformas institucionales y de políticas educativas. Astiz (en este número especial) explica muy bien las premisas principales de este enfoque:

Los modelos culturales internacionales establecen patrones normativos comunes, raramente cuestionados, que determinan no sólo lo que sería lo más apropiado, sino qué políticas se deberían seguir en determinadas circunstancias [...]. Dichos modelos generan un isomorfismo de estructuras y políticas de las cuales los actores raramente pueden escapar. Para esta corriente neo-institucional, los actores están motivados a adoptar determinadas prácticas por una preocupación de estatus. Es decir, en un intento por legitimarse ante sus pares internacionales (otros estados-nación, organizaciones internacionales, u otros colegas que adoptaron las supuestas medidas con anterioridad), los actores nacionales adoptan prácticas o normas que ya poseen reconocimiento global. 
Varios autores, incluyendo Astiz (2006) y Bromley (2016), aplican la Teoría de la Cultura Mundial (WCT, por sus siglas en inglés) para entender la diseminación global del school-based management y de otras políticas de NGP en educación. Según Bromley (2016), para entender las características más importantes de la administración y de la política educativa global cabe adoptar una perspectiva culturalista y, en concreto, poner el acento en procesos globales de racionalización cultural a través de los cuales se define cuál es el comportamiento apropiado y legítimo de los individuos en el contexto de las organizaciones.

La WCT se revela como un marco teórico útil para desafiar enfoques racionalistas y excesivamente historicistas sobre la formación de políticas, en el sentido que aporta elementos conceptuales para entender por qué los países adoptan políticas cuya efectividad no ha sido probada aún (como sucede, de hecho, con muchas de las medidas más emblemáticas de la NGP), o por qué los países introducen políticas globales en marcos institucionales locales en los que dichas medidas, aparentemente, no encajan desde una perspectiva de la tradición institucional vigente. No obstante, este enfoque teórico ha sido criticado por concluir - e incluso celebrar - que un amplio abanico de políticas educativas está convergiendo globalmente, sin reflexionar suficientemente sobre qué intereses y relaciones de poder sustenta dicha convergencia de políticas. (CARNEY; RAPPLEYE; SILOVA, 2012)

Por su parte, aquellos investigadores que estudian la política educativa desde una perspectiva micro critican la WCT por centrarse excesivamente en la adopción formal de las políticas globales. Para ellos, el hecho de que más países converjan en la adopción de un determinado discurso educativo no significa que la convergencia esté teniendo lugar al nivel de las prácticas educativas reales. Más bien, las políticas educativas globales, como las que conforman la doctrina de la NGP, son activamente reinterpretadas y modificadas tanto por los policy-makers nacionales como por los agentes que las aplican en última instancia, como son los profesores y los directores. (ANDERSON-LEVITT, 2003; BALL, 1998) Como pone de manifiesto Astiz en este número, si bien es cierto que políticas de NGP como la autonomía escolar son mundialmente aceptadas como válidas y proveen legitimidad al cambio educativo, "[...] simultáneamente se observa una desviación de dichas normas culturales globales en los procesos de implementación [...]”. En concreto, el estudio comparativo de Astiz sobre la aplicación de medidas a favor de la autonomía escolar en la provincia de Buenos Aires (en este volumen) muestra cómo dicha aplicación se encuentra enraizada en "[...] las instituciones organizativas de los sistemas sociopolíticos que tienden a privilegiar ciertos intereses y desmovilizar otros [...]".

Otra forma de legitimación externa en el campo de las políticas educativas se encuentra en la acción y el modus operandi de las organizaciones internacionales. En el apartado anterior hacíamos referencia al rol jugado por el 
Banco Mundial en la difusión de las políticas de NGP en la década de los noventa, no obstante, actualmente, la OCDE es uno de los principales instigadores de dichas políticas en el campo educativo. A pesar de que la OCDE no es partidaria de políticas de mercado drásticas en educación, sí que se muestra muy favorable a medidas como la autonomía escolar o la rendición de cuentas que, como hemos visto, ocupan un lugar central en el instrumental de política educativa de la NGP. De hecho, después de cinco ediciones del influyente informe PISA (Programa Internacional para la Evaluación de Estudiantes), la autonomía escolar con medidas de rendición de cuentas es una de las recomendaciones más reiteradas por parte de esta organización internacional. Este tipo de recomendaciones tiene una influencia política significativa. Según un estudio reciente, 29 representantes de los países de la OCDE (de un total de 37) admitieron que las recomendaciones provenientes del informe PISA han influido de forma directa en los procesos de formulación de políticas sobre rendición de cuentas a nivel nacional. (BREAKSPEAR, 2012)

Varios de los artículos incluidos en este número especial corroboran el rol clave que la OCDE juega en la legitimación de reformas educativas de NGP. Por ejemplo, la OCDE llevó a cabo en Noruega en 1998 una revisión de la política educativa que puso de relieve un nivel insuficiente de control estatal sobre el sistema educativo. Como consecuencia de dicha revisión, Noruega introdujo un sistema centralizado para la supervisión de los resultados educativos. Más adelante, el lanzamiento del primer informe PISA de la OCDE, en el que Noruega obtuvo resultados muy por debajo sus expectativas, hizo que "los logros académicos de los estudiantes devinieran el centro de atención” y "[...] el discurso entorno a la necesidad de que los profesores rindan cuentas se volvió más persistente y agresivo [...]”. (MOLLER; SKEDSMO en este número)

Algo similar ha sido observado en Cataluña, donde el informe PISA se ha convertido en un referente de reforma educativa clave. PISA, por un lado, ha hecho que los diseñadores de políticas catalanes sean más conscientes del problema de calidad educativa que afrontan y, por el otro, les ha ayudado a identificar y 'tomar prestadas' soluciones de política educativa como la autonomía escolar y el liderazgo escolar. (véase VERGER; CURRAN; PARCERISA en este número) En Italia, las ideas y herramientas de la NGP han sido adoptadas por economistas y políticos con vínculos al Banco Central Europeo y a la OCDE. En su momento, este país fue también sacudido por el informe PISA, el cual definía el sistema educativo italiano como inefectivo y de bajo rendimiento. (ver GRIMALDI; SERPIERI; TAGLIETTI en este número)

Finalmente, muchos gobiernos nacionales han concebido e instrumentalizado los resultados de PISA como una oportunidad política con la que legitimar sus preferencias de políticas pre-establecidas (MARTENS et al., 2010), y justificar así reformas educativas que, de lo contrario, hubieran encontrado más resistencia. (GREK, 2007) Francia representa un buen ejemplo de cómo opera 
este mecanismo. En este país, la configuración del sistema de evaluación educativa ha sido justificada en base a estudios e informes realizados por la OCDE, mientras que el informe Pisa ha legitimado la adopción de una política de competencias que encaja con las preferencias del gobierno francés. (ver DEROUET; NORMAND; PACHECO en este número)

\subsection{El rol de las ideas: las críticas al profesorado y la emergencia de un 'nuevo profesionalismo'}

Desde posiciones teóricas de corte racionalista, las políticas de la NGP en educación, como la autonomía escolar, los incentivos docentes basados en el rendimiento, o la elección de centro, se estarían extendiendo globalmente porque la evidencia pone de manifiesto que estas políticas funcionan o han funcionado bien en muchos lugares. Sin embargo, las soluciones de NGP en educación no son adoptadas necesariamente porque "funcionan" (de hecho, hay muchos interrogantes empíricos todavía abiertos al respecto), sino porque existe una percepción generalizada de que son políticas que podrían solucionar una parte importante de los problemas más apremiantes de los sistemas educativos contemporáneos. Factores de carácter discursivo e ideacional se encuentran, en gran medida, detrás de esta percepción social tan generalizada. Por lo tanto, este tipo de factores necesitan ser vistos también como fuerzas subyacentes a la expansión de las reformas basadas en la NGP.

Las 'ideas' influyen en procesos de toma de decisiones sobre políticas educativas de diferentes maneras. Los marcos ideacionales dan forma a las percepciones de los policy-makers, les proporcionan lógicas para la acción e interpretaciones del mundo exterior. Las ideas, en su rol de 'filtros cognitivos', son especialmente relevantes en el contexto de ámbitos de gran complejidad y donde prevalece la incertidumbre, como es claramente el caso de la educación. (KJAER; PEDERSEN, 2001) En general, a la hora de entender el poder de ideas como la NGP en el campo educativo, resulta necesario considerar que la NGP, en sí misma, funciona como un poderoso 'paradigma de políticas' (cf. HALL, 1993) que dota a los policy-makers de marcos interpretativos, categorías y modelos de política que contribuyen a reducir la incertidumbre habitual a la hora de tomar decisiones sobre reforma educativa o mejora escolar.

David Hall y Helen Gunter (en este número) exponen cómo la materialización de las ideas de la Nueva Derecha en la política educativa del Reino Unido generó un cambio de paradigma en las políticas de bienestar que allanó el camino de los gobiernos del Partido Laborista, los cuales construyeron su política educativa sobre los marcos de referencia establecidos en los años ochenta. Sin embargo, más allá de los paradigmas de políticas, otros tipos de ideas como pueden ser la 
opinión pública o los sentimientos de la población son también muy importantes cuando se trata de entender la expansión global de la NGP. Ello, en parte, se debe al hecho de que, como Kalimullah et al. $(2012,19)$ plantean, "[...] la burocracia hoy día tiene pocos partidarios en cualquier sitio. Cualquier solución que ofrezca una reducción en burocracia es de esperar que sea popular [...]”.

El hecho de que algunas políticas de NGP, como la autonomía escolar, funcionen como un 'significante flotante' es también importante para entender la hegemonía de las soluciones de NGP en la agenda educativa global desde un punto de vista discursivo. La 'autonomía escolar' es un concepto lo suficientemente ambiguo como para generar aceptación entre un amplio abanico de audiencias e ideologías. Como muestran Verger, Curran y Parcerisa (en este volumen), los socialdemócratas ven la autonomía como una forma de promover el sector público y de hacerlo más efectivo; los neoliberales la consideran una manera de empequeñecer el aparato estatal y de introducir lógicas de mercado en el sector público; los conservadores la ven como una vía para profesionalizar y jerarquizar la gestión de los centros educativos; y, finalmente, los educadores progresistas la consideran como una vía de introducir pedagogías renovadoras, y una educación más significativa y adaptada al contexto.

Los autores constructivistas consideran que la persuasión, la deliberación y la acción comunicativa actúan como factores causales del comportamiento social y del cambio institucional. (RISSE, 2000) Para los constructivistas, la cuestión principal es entender los procesos por los cuales ideas inicialmente minoritarias devienen ampliamente aceptadas e institucionalizadas. (HASENCLEVER et al., 1996) Como desarrollaremos a continuación, los estudios sobre NGP representan un campo de investigación apropiado para explorar cómo grupos de expertos, comunidades epistémicas, advocacy coalitions y emprendedores de políticas han promovido entre los decisores de políticas cuáles son los problemas clave en los que tienen que fijarse, así como las soluciones políticas más efectivas.

Desde la década de los 1980, este tipo de actores ha dirigido numerosas críticas a la profesión docente, en ocasiones por su proximidad a la izquierda activista o reformista, pero también porque considera a los docentes responsables del descenso de estándares de calidad y de no aceptar una educación de corte más tradicional. Intelectuales, políticos y Think Tanks difundieron con éxito la idea de que es urgente reestructurar la profesión docente, al tiempo que representantes de la esfera de los negocios criticaban al sistema educativo por su falta de efectividad económica y de adaptación al mercado de trabajo. Estas críticas fueron ampliadas con acusaciones de corporativismo contra una profesión que se consideraba que defendía angostos intereses y privilegios mientras el resto de la sociedad realizaba sacrificios para lidiar con consecutivas crisis económicas. Al tiempo que el sindicalismo y los movimientos sociales se estaban debilitando, tanto la representación sindical como la autonomía profesional eran alteradas en nombre de la necesidad 
de aplicar reformas sobre competencias curriculares y rendición de cuentas. La lógica de la gestión penetraba en los sistemas educativos, y se corría el riesgo de reducir el espíritu colegial y la solidaridad entre los profesores. Si bien algunos profesores resistieron la doctrina del gerencialismo, otros adoptaron estos nuevos estándares para obtener ventajas salariales, bonus y primas, o para acceder a nuevos puestos y carreras profesionales.

Como demuestra Hirschmann (1991), la retórica de la Nueva Derecha ha sido muy incisiva a la hora de criticar las ideas progresistas sobre el sector público desde los marcos de significado de la perversidad, la futilidad y el peligro. Según el marco de la perversidad, el principio de la igualdad ha incrementado la burocracia y ha disminuido los estándares de calidad. Los sistemas educativos, debido al proceso de democratización y la ampliación del número de profesores y funcionarios, ha hecho aumentar el gasto público de manera insostenible. El número creciente de estudiantes ha conllevado la devaluación de los títulos académicos y la disminución de los estándares curriculares. Desde el marco de la futilidad se asume que los estudiantes desfavorecidos no han mejorado sus resultados, ni siquiera en zonas de educación prioritaria, a pesar del aumento en el gasto público. Dichos argumentos invocan el fracaso de los estudiantes en la adquisición de competencias, el aumento del abandono escolar o los débiles índices de inclusión entre los graduados. Finalmente, la retórica del peligro considera que la igualación de las condiciones de enseñanza ha amenazado la libertad de elección y los valores educativos tradicionales.

Este tipo de críticas contribuyen a deslegitimar la profesión docente y el rol que los docentes deberían jugar a la hora de democratizar el acceso a la escolarización y al conocimiento. El profesorado es estigmatizado como poco innovador y resistente al cambio. El sindicalismo y la representación colegial son percibidas como un déficit democrático basado en relaciones de influencia. Valores e ideales de autonomía profesional basados en el compañerismo, la lealtad y la solidaridad, son desafiados en nombre de la motivación individual, el éxito y el desarrollo de proyectos y del liderazgo. Un nuevo régimen de rendición de cuentas y de técnicas y herramientas de gestión - consideradas como objetivas, adaptables y flexibles es así introducido con el objetivo de la mejora escolar, la búsqueda de la excelencia y de poner freno a las estrategias corporativistas. (THRUPP; WILMOTT, 2003)

\section{Discusión: sobre las múltiples trayectorias (y paradojas) de la NGP en educación}

La NGP es una constelación de teorías gerenciales, marcos y herramientas, reglamentos y normas para la reforma del sector público producidas por redes transnacionales de expertos, policy-makers, organizaciones internacionales, think 
tanks, fundaciones y grupos de consultoría. Sus discursos e instrumentos circulan a nivel global, pero son reformulados y traducidos en contextos nacionales y locales de tal manera que las políticas finalmente retenidas pueden tener aproximaciones bien diferenciadas. Así pues, a la hora de adoptar la NGP, algunos países hacen más hincapié en el mercado y la privatización, mientras que otros están más preocupados por la descentralización y la rendición de cuentas. La penetración de la ideología gerencial como mecanismo de garantía de la calidad varía de un contexto nacional a otro. También ha creado nuevos desafíos para la profesión docente, incluyendo nuevas normas, dispositivos e instrumentos para la rendición de cuentas que se integran en el día a día del trabajo docente. Todo ello con el agravante de que los maestros no tienden a ser consultados cuando se definen los estándares de calidad y los criterios de evaluación de sus estudiantes en pruebas estandarizadas.

Ahora bien, la transformación de la organización escolar bajo la NGP tiene el potencial de dar más flexibilidad y autonomía a los maestros: libera iniciativas y proyectos, enfatiza la responsabilidad individual y el nuevo profesionalismo. La NGP ha combinado las tecnologías del taylorismo y la burocracia con nuevas tecnologías gerenciales, y con ello ha generado un resultado aparentemente contradictorio. Por un lado, los maestros tienen que aplicar reglas y cumplir objetivos que les vienen dados por los administradores del sistema de forma jerárquica. (GEWIRTZ, 2002) Por otro lado, el nuevo gerencialismo hace hincapié en el trabajo en equipo, la flexibilidad, el liderazgo compartido, y el desarrollo profesional, medidas que ofrecen oportunidades para empoderar a los maestros y reconocer sus iniciativas individuales (mientras estén, eso sí, al servicio de un mayor rendimiento educativo). El trabajo y el espíritu colectivos son alentados por el desarrollo del individualismo, a la vez que la intensificación del trabajo y la fragmentación de la carrea docente tiene el potencial de socavar la cooperación y la colegialidad en las escuelas.

No obstante, es todavía pronto para sacar conclusiones generalizables sobre los efectos de la NGP. La aplicación e impacto de la NGP está fuertemente condicionada por las tradiciones institucionales y las culturas profesionales que prevalecen en diferentes contextos. Como se observa en los diferentes artículos de este número, el legado del Estado burocrático persiste en la mayoría de países, incluso cuando la administración ha sido reestructurada y progresivamente reconducida hacia prácticas organizativas más fragmentarias y flexibles. La autonomía y la descentralización son combinadas con ideas preconcebidas de la comunidad educativa y/o de los poderes locales sobre cuestiones de gestión escolar. El profesionalismo de los maestros adopta diferentes significados en función de las disputas políticas y tradiciones educativas vigentes. Como resultado de todo ello, se pueden forjar nuevas identidades profesionales y alterarse el equilibrio de poderes en el sistema educativo. La rendición de cuentas, por su parte, puede ser aceptada como un principio de responsabilidad hacia la comunidad y los sectores más vulnerables 
de la sociedad, y no sólo hacia el cliente o el consumidor. En general, cada uno de los instrumentos que conforman el programa de la NGP tiene su propia historia, trayectoria y desarrollo, y es altamente dependiente de contingencias y tradiciones locales, así como del sector concreto en que ésta es adoptada.

Si la NGP es un efecto directo de la globalización, es necesario tener en cuenta algunas traducciones e hibridaciones locales en su aplicación. En Noruega, como hemos visto, el legado de la escuela comprehensiva y el apego al valor de la equidad han mantenido la competencia escolar y la lógica del mercado lejos del campo educativo. (MOLLER; SKEDSMO, en este volumen) El valor de la igualdad también está considerablemente presente en el sistema educativo francés, pero la reforma de la NGP es una fuente de conflictos recurrente entre los partidos políticos y los sindicatos. (ver DEROUET; NORMAND; PACHECO en este número)

Conflictos similares son observables también en Italia, pero con efectos más tangibles ya que, en este país, el nivel de descentralización educativa es más acusado y la penetración de la NGP ha facilitado una mayor apertura a la mercantilización del sistema educativo. (GRIMALDI; SERPIERI; TAGLIETTI en este número) En definitiva, como sostiene Andrade en este número especial, tener una teoría más sofisticada del Estado es condición necesaria para entender los procesos de recontextualización de las reformas de la NGP. Según esta autora, en función del equilibrio de poder y la lucha por la hegemonía dentro del Estado, los resultados de las reformas educativas pueden ser más favorables a los trabajadores y a los grupos vulnerables o, por el contrario, beneficiar a los grupos más privilegiados.

Todo ello refuerza la premisa de que la NGP no es un programa de reforma educativa necesariamente coherente ni uniforme. Sin necesidad de ir más lejos, los estudios de caso recogidos en este monográfico muestran que la aplicación de la NGP en sistemas educativos concretos conlleva una cierta hibridación de políticas y la aplicación de medidas que, a pesar de que interpelan a conceptos muy similares, pueden tener, en esencia, una naturaleza muy diferente. A modo de síntesis de esta idea, la Tabla 2 identifica diferentes configuraciones posibles de las políticas de NGP en educación. ${ }^{2}$ Como se observa en la tabla (concretamente, en la segunda columna), la NGP puede traducirse en opciones de políticas y en intervenciones educativas con diseños muy variados y, en ocasiones, incluso antagónicos. Esto último dificulta todavía más la posibilidad de sacar conclusiones generalizables sobre el impacto de la NGP ya que las políticas de NGP, en función de la configuración concreta que acaben adoptando, tendrán implicaciones muy diferentes en términos de equidad educativa, mercantilización de los servicios públicos, (des)profesionalización docente y/o democracia escolar.

\section{Tabla 2}


Las posibles configuraciones de la NGP en la educación

\begin{tabular}{|c|c|}
\hline POLÍTICAS EDUCATIVAS DE NGP & POSIBLES CONFIGURACIONES \\
\hline $\begin{array}{l}\text { Profesionalización/empoderamiento de los } \\
\text { directores de escuela }\end{array}$ & $\begin{array}{l}\text { - Estilo de dirección jerárquico vs liderazgo } \\
\text { compartido }\end{array}$ \\
\hline $\begin{array}{l}\text { Definición de estándares curriculares } \\
\text { comunes e indicadores de calidad y de } \\
\text { benchmarks sobre "éxito" educativo }\end{array}$ & $\begin{array}{l}\text { - Diferentes niveles de estandarización y } \\
\text { prescripción de los contenidos y } \\
\text { metodologías de enseñanza } \\
\text { - Presencia o ausencia de la equidad como } \\
\text { una condición de calidad/éxito educativo }\end{array}$ \\
\hline $\begin{array}{l}\text { Evaluación externa de los resultados y del } \\
\text { rendimiento escolar }\end{array}$ & $\begin{array}{l}\text { - Evaluación sumativa } v s \text { formativa } \\
\text { - Presencia de incentivos materiales, } \\
\text { formativos o simbólicos vinculados a los } \\
\text { resultados de la evaluación } \\
\text { - Publicación o no publicación de los } \\
\text { resultados obtenidos por las escuelas en } \\
\text { pruebas estandarizadas }\end{array}$ \\
\hline $\begin{array}{l}\text { Autonomía escolar; school-based } \\
\text { management }\end{array}$ & $\begin{array}{l}\text { - Foco de la autonomía en la gestión de } \\
\text { recursos, en la organización escolar y/o en } \\
\text { los asuntos pedagógicos } \\
\text { - Locus de la autonomía: el director vs la } \\
\text { comunidad escolar }\end{array}$ \\
\hline $\begin{array}{l}\text { Subsidios públicos a las escuelas privadas; } \\
\text { financiación per cápita o competitiva }\end{array}$ & $\begin{array}{l}\text { - El tipo de competencia entre escuelas } \\
\text { (resultante de la financiación per cápita) } \\
\text { estará condicionada por los niveles de } \\
\text { regulación y control de la gratuidad } \\
\text { educativa, del acceso a las escuelas y de la } \\
\text { elección escolar }\end{array}$ \\
\hline $\begin{array}{l}\text { Empoderamiento de las familias/clientes en } \\
\text { los servicios públicos }\end{array}$ & $\begin{array}{l}\text { - Diferentes niveles y ámbitos de } \\
\text { participación de las familias (gestión, } \\
\text { presupuesto, cuestiones educativas, servicios } \\
\text { periféricos) } \\
\text { - Dar más voz a las familias en las escuelas } \\
\text { (para que puedan transmitir sus demandas y } \\
\text { preocupaciones al profesorado), o fomentar } \\
\text { que opten por la elección/salida de las } \\
\text { escuelas (dinámica de mercado) } \\
\end{array}$ \\
\hline $\begin{array}{l}\text { Financiación de las escuelas y/o docentes en } \\
\text { base a criterios de mérito/productividad }\end{array}$ & $\begin{array}{l}\text { - Evaluaciones de los docentes a nivel } \\
\text { individual o de escuela } \\
\text {-Evaluación de los docentes basada en su } \\
\text { dominio del currículum, en los resultados de } \\
\text { sus estudiantes, o en los procesos de } \\
\text { enseñanza }\end{array}$ \\
\hline
\end{tabular}

Fuente: Autores.

Este monográfico reflexiona sobre las múltiples trayectorias de las políticas de NGP en realidades educativas concretas, pero también sobre el carácter paradójico que a menudo adoptan dichas trayectorias. Por regla general, las políticas globales, dada su naturaleza inherentemente desterritorializada, afrontan 
importantes desafíos una vez son adoptadas e implementadas en marcos institucionales y políticos concretos. Ahora bien, la NGP es probablemente uno de los modelos de reforma educativa global que refleja más contradicciones y tensiones a la hora de transitar de la teoría a la práctica, o de lo global a lo local. De hecho, la mayoría de los estudios de caso recogidos en este número especial identifican algún tipo de paradoja (o de desviación respecto a los preceptos iniciales) en el tránsito que la NGP realiza hacia las prácticas educativas concretas. Por ejemplo:

- En Noruega, las reformas de la NGP se han diseñado para desburocratizar los servicios públicos, pero han terminado generando más carga burocrática para el personal docente.

- En Cataluña, la adopción de políticas de NGP se justifica sobre la base de las recomendaciones de la OCDE y de PISA, pero estas recomendaciones son a menudo alteradas para hacerlas encajar mejor en les preconcepciones y preferencias de los policy-makers locales.

- En Francia, el apego social a la igualdad y al currículo nacional han acelerado el cambio hacia una política de estándares y de competencias básicas, mientras que las medidas de descentralización y autonomía de centro han quedado más bien en un segundo plano.

- En el Reino Unido, el desarrollo de la NGP por parte del partido laborista no ha representado una alternativa al paradigma de la nueva derecha instaurado por el gobierno de Thatcher, sino que ha promovido más bien la mercantilización y la privatización de la educación pública.

- En Quebec, la política de rendición de cuentas ha fortalecido la regulación estatal burocrática, al mismo tiempo que ha mantenido el poder de las comunidades locales y ha ampliado las posibilidades de elección de escuela.

- En Chile, a diferencia de la mayoría de las experiencias internacionales (donde la NGP ha sido adoptada como una forma de mercantilizar la educación), la NGP ha sido concebida como una manera de controlar las fuerzas del mercado en educación, así como sus efectos perversos en términos de desigualdades educativas y segregación escolar (aunque la evidencia existente no indica que haya conseguido paliar dichos efectos de forma significativa).

Para acabar, quisiéramos destacar que este monográfico, más allá de recoger un conjunto importante de estudios de caso de gran rigor sobre la adopción y la recontextualización de políticas de NGP en educación, debe leerse como una invitación a seguir explorando e investigando la relación multifacética - y a menudo paradójica - entre la NGP, como modelo global de reforma del sector público, 
y las transformaciones educativas que, en la actualidad, se están produciendo en múltiples puntos del planeta y a diferentes escalas.

\author{
Antoni Verger* \\ Romuald Normand ${ }^{*}$ \\ Organizadores
}

\title{
Notas
}

1. Los coordinadores de este monográfico quisieran agradecer a Ramón Pacheco y a Lluís Parcerisa su apoyo y aportaciones a la edición de este volumen y a la elaboración de este artículo introductorio en particular.

2. Nótese que la columna sobre 'políticas educativas' de la Tabla 2 comparte la mayoría de los contenidos con la Tabla 1 de este mismo artículo.

\section{Referencias bibliográficas}

ANDERSON-LEVITT, K. M. Local Meanings, Global Schooling: Anthropology and world culture theory. Basingstoke: Palgrave Macmillan, 2003.

ASTIZ, M. F. School autonomy in the province of Buenos Aires, Argentina: Evidence from two school districts. Comparative Education, v. 42, n. 2, 2006, p. 203-223.

BALL, S. J. Big policies/small world: An introduction to international perspectives in education policy. Comparative Education, v. 34, n. 2, 1998, p. 119-130.

. Performativities and fabrications in the education economy: towards the performative society. In: GLEESON, D.; HUSBANDS, C. (Eds.). The Performing School. London: Routledge Falmer, 2001, p. 210-225.

. The teacher's soul and the terrors of performativity. Journal of education policy, v. 18 , n. 2, 2003, p. 215-228.

BROMLEY, P. Policy and Administration as Culture: Organizational Sociology and Cross-National Education Trends. In: MUNDY, K. et al. Global Education Policy Handbook. London: Wiley-Blackwell. 2016-forthcoming.

CALLAHAN, R. E. Education and the Cult of Efficiency: Study of the Social Forces that have shaped the Administration of the Public Schools. Chicago: University of Chicago Press, 1962.

\footnotetext{
* Universitat Autònoma de Barcelona, Departamento de Sociología. Barcelona, Cataluña, España. E-mail de contacto: tverger@gmail.com.

** Université de Strasbourg, Faculté des sciences sociales, Sociétés, Acteurs et Gouvernement en Europe (Sage). Strasbourg, France.
} 
CAMPBELL, J. L. Institutional change and globalization. Princeton, NJ: Princeton University Press, 2004.

CARNEY, S.; RAPPLEYE, J.; SILOVA, I. Between Faith and Science: World Culture Theory and Comparative Education. Comparative Education Review, v. 56, n. 3, 2012, p. 366-393.

CHRISTENSEN, T.; L/EGREID, P. Beyond NPM? Some Development Features. In: . (Eds.). The Ashgate Research Companion to New Public Management. Farnham, England: Ashgate Publishing Ltd., 2011, p. 391-403.

CLARKE, J.; NEWMAN, J. The Managerial State. London: Sage, 1997.

EURYDICE EUROPEAN UNIT. School autonomy in Europe policies and measures. Brussels: Eurydice, 2007.

EVETTS, J. The sociological analysis of professionalism occupational change in the modern world. International sociology, v. 18, n. 2, 2003, p. 395-415.

FINE, B. The new development economics: after the Washington consensus. London: Zed Books, 2006.

GEWIRTZ, S. The Managerial School: Post-welfarism and Social Justice in Education. Buckingham: Open University Press, 2002.

GREK, S. Governing by numbers: The PISA 'effect' in Europe. Journal of Education Policy, v. 24, n. 1, 2009, p. 23-37.

GUNTER, H.; FITZGERALD, T. New Public Management and the modernisation of education systems 1. Journal of Educational Administration and History, v. 45, n. 3, 2013, p. 213-219.

GUNTER, H. M.; FORRESTER, G. School leadership and education policy-making in England. Policy Studies, v. 30, n. 5, 2009, p. 495-511.

HALL, P. Policy Paradigms, Social Learning and the State. The Case of Economic Policymaking in Britain. Comparative Politics, v. 25, n. 3, 1993, p. 275-296.

HASENCLEVER, A.; MAYER, P.; RITTBERGER, V. Interests, Power, Knowledge: The Study of International Regimes. Mershon International Studies Review, v. 40, 1996, p. $177-228$.

HENDRIKS, F.; TOPS, P. Local public management reforms in the Netherlands: Fads, fashions and winds of change. Public Administration, v. 81, n. 2, 2003, p. 301-323.

HIRSCHMANN, A. O. The Rhetoric of Reaction: Perversity, Futility, Jeopardy. Cambridge, MA: The Belknap Press of Harvard University Press, 1991.

HOOD, C. A public management for all seasons? Public Administration, v. 69, n. 1, 1991, p. 3-19.

HOOD, C.; PETERS, G. The middle aging of new public management: Into the age of paradox? Journal of Public Administration Research and Theory, v. 14, n 3, 2004, p. 267-282. 
HUDSON, C. Governing the governance of education: The state strikes back? European Educational Research Journal, v. 6, n. 3, 2007, p. 266-282.

KALIMULLAH, N. A.; ASHRAF, K. M.; ASHADUZZAMAN, M. N. New public management: Emergence and principles. BUP Journal, v. 1, n. 1, 2012, p. 1-22.

KJAER, P.; O. K., PEDERSEN. Translation liberalization: Neoliberalism in the Danish negotiated economy. In: CAMPBELL, J. L.; PEDERSEN, O. K. (Eds.). The Rise of Neoliberalism and Institutional Analysis. Princeton: Princeton University Press, 2001, p. 219-248.

KLITGAARD, M. B. Why are they doing it? Social democracy and market-oriented welfare state reforms. West European Politics, v. 30, n.1, 2007, p.172-194.

MAROY, C.; VOISIN, A. As transformaçôes recentes das políticas de accountability na educação: desafios e incidências das ferramentas de ação pública. Educ. Soc., v. 34, n. 124, 2013.

MARTENS, K. et al. Transformation of education policy. The impact of the bologna process and the PISA study in comparative perspective. Basingstoke: Palgrave-Macmillan, 2010.

MCLAUGHLIN, K.; OSBORNE, S. P.; FERLIE, E. New public management: Current trends and future prospects. New York, NY: Routledge, 2001.

OECD. School autonomy and accountability: Are they related to student performance? oct. 2011. [Pisa in Focus 9]. Fuente: <http://www.oecd.org/pisa/pisaproducts/pisainfocus/48910490.pdf>.

. Synergies for Better Learning: An International Perspective on Evaluation and Assessment. Francia: OECD, 2013, 672p. Fuente: <http://www.oecd.org/edu/school/ Synergies_for_Better_Learning_summary_Spanish.pdf $>$.

OSBORNE, D.; GAEBLER, T. Reinventing government: How the entrepreneurial spirit is transforming the public sector. New York: Plume, 1993.

PECK, J.; THEODORE, N. Mobilizing policy: models, methods, and mutations. Geoforum, v. 41, n. 2, 2010, p. 169-74.

POWER, M. The Audit Society. London: Demos, 1997.

RISSE, T. Let's Argue! Communicative Action in World Politics. International Organization, v. 54, p. 1-39, 2000.

SLEEGERS, P.; WESSELINGH, A. Dutch dilemmas: Decentralisation, school autonomy and professionalisation of teachers. Educational Review, v. 47, n. 2, 1995, p. 199-207.

THRUPP, M.; WILLMOTT, R. Education Management in Managerialist Times. Beyond the Textual Apologists. Buckingham: Open University Press, 2003.

TOLOFARI, S. New public management and education. Policy Futures in Education, v. 3, n. 1, 2005, p. 75-89.

TYACK, D.; HANSOT, H. Managers of virtue: public school leadership in America, 1820-1980. New York: Basic Books, 1982. 
VERGER, A.; CURRAN, M. New public management as a global education policy: its adoption and re-contextualization in a Southern European setting. Critical Studies in Education, v. 55, n. 3, 2014, p. 253-271.

VERGER, A.; NOVELLI, M.; ALTINYELKEN, H. K. Global Education Policy and International Development: New Agendas, Issues and Policies. London: Bloomsbury, 2012, p. 3-32.

VIGODA, E. New public management. In: Rabin. J. (Ed.). Encyclopedia of public administration and public policy. v. 2. New York: Marcel Dekker, 2003.

WIBORG, S. Neo-liberalism and universal state education: The cases of Denmark, Norway and Sweden 1980-2011. Comparative Education, v. 49, n. 4, 2013, p. 407-423.

Recebido em 18 de agosto de 2015.

Aprovado em 28 de setembro de 2015.

DOI: http://dx.doi.org/10.1590/ES0101-73302015152799 